\title{
Development of Formaldehyde Gas Sensor that Exhibits Distinct Color Changes
}

\author{
Yasumasa Kanekiyo, ${ }^{1 *}$ You Mitani, ${ }^{1}$ Miho Suda,${ }^{1}$ Hiroshi Aoki ${ }^{2}$ and Hironobu Minai ${ }^{3}$ \\ ${ }^{1}$ Kitami Institute of Technology, 165 Koen-Cho, Kitami, Hokkaido 090-8507, Japan \\ ${ }^{2}$ National Institute of Advanced Industrial Science and Technology (AIST), \\ 16-1 Onogawa, Tsukuba, Ibaraki 305-8569, Japan \\ ${ }^{3}$ JOKOH Co., Ltd., 3-19-4 Hongo, Bunkyo-ku, Tokyo 113-0033, Japan
}

(Received December 15, 2019; accepted February 21, 2020)

Keywords: formaldehyde, sensor, colorimetric, film, dye

A new type of colorimetric sensor that detects formaldehyde in air was developed. The sensor consists of a thin film containing primary amino groups on a glass substrate. After exposure to gaseous formaldehyde, the thin film was immersed in an aqueous solution containing an anionic dye. The color of the thin film distinctly changed in accordance with the formaldehyde concentration in air. It was found that the sensitivity of this sensor is significantly affected by factors such as the exposure time, the humidity of air, and the monomer composition of the thin film.

\section{Introduction}

Formaldehyde is widely used in household products such as furniture glues, inks, and sterilizers. In recent years, it has been recognized as a major cause of "sick house syndrome" or "sick building syndrome". (1-4) To reduce such health risks, it is necessary to provide a sensitive, durable, and easily operable sensor for formaldehyde at a low cost.

To date, a variety of sensing methods for formaldehyde including chromatography, ${ }^{(5)}$ spectroscopy, ${ }^{(6)}$ and electrochemistry ${ }^{(7,8)}$ have been reported. These methods enabled formaldehyde measurement at the ppb level. However, their application for personal use is problematic because they require expensive devices, complex operations, or a long measurement time. Alternatively, colorimetric sensors for formaldehyde have been developed in recent years. ${ }^{(9-12)}$ Colorimetric methods are advantageous for the development of sensors for personal use because of their easy handling, portability, and no requirement of special devices. Previously reported colorimetric sensors were based on direct reactions of formaldehyde with dyes or coloring reagents. In these cases, the available dyes and coloring reagents are limited to those that react with formaldehyde while undergoing color changes. Therefore, the choice of colors is limited, and it is difficult to create a variety of color changes in the sensors. Another problem is that the dyes and coloring reagents are often toxic and harmful to users.

*Corresponding author: e-mail: kanekiyo@mail.kitami-it.ac.jp

https://doi.org/10.18494/SAM.2020.2738 
In recent years, we have been developing colorimetric sensors that show a variety of distinct color changes in thin films. ${ }^{(13-16)}$ The color change mechanisms of these sensors were based on changes in the charge state of thin films caused by reactions with target molecules. These changes resulted in the absorption or desorption of anionic or cationic dyes due to changes in the electrostatic interactions. Previously, we reported a novel sensor for detecting formaldehyde in aqueous solutions that uses the ability of a primary amino group to react with formaldehyde, resulting in the formation of an imine (Schiff base) via a dehydration reaction. ${ }^{(17)}$ Before the reaction with formaldehyde, the primary amino group was positively charged owing to protonation. Hence, the thin film was colored by the adsorption of anionic dyes via electrostatic interaction. After immersion in formaldehyde solutions, the anionic dyes were desorbed from the thin films, and thus the color of the thin films changed with increasing formaldehyde concentration. This color change was considered to be due to the conversion of the positively charged primary amino group to the neutral imine group. Accordingly, we succeeded in measuring formaldehyde in the concentration range of $0-100 \mathrm{mM}$ in an aqueous solution with a detection limit of $3 \mathrm{mM}$.

We herein report our preliminary results for the sensing of formaldehyde in air. The preparation method of the sensor was basically the same as that in our previous report. First, the sensor was exposed to gaseous formaldehyde, and then the thin film was colored with an anionic dye by immersing it in an aqueous dye solution. The effects of the exposure time to formaldehyde, the humidity of air, and the monomer composition of the thin film were investigated.

\section{Materials and Methods}

\subsection{Materials}

Primary amine monomer 1 [ $N$-(3-aminopropyl)methacrylamide hydrochloride], acrylamide $\mathbf{2}$, crosslinker 3 ( $N, N^{\prime}$-methylene-bis-acrylamide), initiator 4 [2,2'-azobis(2-methylpropionamidine) dihydrochloride], anionic dye 5 (Fast Green FCF), paraformaldehyde, sodium metaborate tetrahydrate, boric acid, sodium carbonate, and dimethyl sulfoxide (DMSO) were purchased from FUJIFILM Wako Pure Chemical Corporation (Osaka, Japan). The chemical structures of these compounds are shown in Fig. 1. All chemicals were of the reagent grade and were used as received without further purification. Pattern-printed microscope slides were obtained from Matsunami Glass Industries (Osaka, Japan), which are glass plates covered with a hydrophobic polymer layer ( $20 \mathrm{~mm}$ in thickness) with circular holes ( $8 \mathrm{~mm}$ in diameter), leaving the glass surface exposed.

\subsection{Preparation of sensors}

The formaldehyde-responsive thin films were prepared on a pattern-printed microscope slide by the radical copolymerization of primary amine monomer $\mathbf{1}$, acrylamide $\mathbf{2}$, and crosslinker $\mathbf{3}$ in the presence of initiator 4. Monomer compositions for the preparation of the thin films are 
<smiles>C=CC(N)=O</smiles>

1<smiles>C=COCNCNC(=O)C=C</smiles>

3<smiles>CC(C)(N=NC(C)(C)C(N)=NCl)C(=N)Cl</smiles>

4<smiles>CCN(Cc1cccc(S(=O)(=O)[O-])c1)c1ccc(C(=C2C=CC(=NC(=O)c3cccc([S+](=O)([O-])[O-])c3)C=C2)c2ccc(O)cc2[S+](=O)[O-])cc1</smiles>

5

Fig. 1. Chemical structures of compounds used in this study.

Table 1

Monomer compositions for film synthesis.

\begin{tabular}{lccccc}
\hline & $\mathbf{1}$ & $\mathbf{2}$ & $\mathbf{3}$ & $\mathbf{4}$ & Solvent \\
\hline Sample A & $120 \mathrm{mmol}$ & $680 \mathrm{mmol}$ & $40 \mathrm{mmol}$ & $60 \mathrm{mmol}$ & $\mathrm{H}_{2} \mathrm{O} 840 \mu \mathrm{L} / \mathrm{DMSO} 80 \mu \mathrm{L}$ \\
Sample B & $200 \mathrm{mmol}$ & $600 \mathrm{mmol}$ & $40 \mathrm{mmol}$ & $60 \mathrm{mmol}$ & $\mathrm{H}_{2} \mathrm{O} 840 \mu \mathrm{L} / \mathrm{DMSO} 80 \mu \mathrm{L}$ \\
\hline
\end{tabular}

shown in Table 1. The monomer solutions $(2.3 \mu \mathrm{L}$ each) were poured onto the glass surface of a pattern-printed microscope slide by micropipetting and covered with an acrylic plate. Polymerization was conducted by irradiating the monomer solutions sandwiched between the microscope slide and the acrylic plate with UV light $(365 \mathrm{~nm})$ at room temperature for $3 \mathrm{~h}$. To avoid interference from oxygen, the procedure was conducted under nitrogen atmosphere in a glove box. The acrylic plate was then removed from the microscope slide, and the resulting thin films on the slide were washed with water and then dried in air. After dividing the plate, each circular spot was immersed in aqueous sodium carbonate solution $(0.1 \mathrm{M})$ while stirring at $25{ }^{\circ} \mathrm{C}$ for $48 \mathrm{~h}$. Then, the thin film surface was washed with water and then dried under vacuum.

\subsection{Method of sensing}

A gas cylinder containing purified air was connected to a gas washing bottle and a threenecked flask through plastic tubes as shown in Fig. 2. The formaldehyde sensor was set at the bottom of the flask, and an aqueous formaldehyde solution was poured in the gas washing bottle, through which the purified air was bubbled at a rate of $100 \mathrm{~mL} \mathrm{~min}{ }^{-1}$. The formaldehyde concentration in the air was determined according to Henry's equation:

$$
P_{\mathrm{HCHO}}=C_{\mathrm{HCHO}} \times H
$$

where $P_{\mathrm{HCHO}}$ is the vapor pressure of formaldehyde (atm), $C_{\mathrm{HCHO}}$ is the concentration of formaldehyde in solution (mol L ${ }^{-1}$ ), and $H$ is Henry's constant $\left(2.6 \times 10^{-4} \mathrm{~L} \mathrm{~atm} \mathrm{~mol}^{-1}\right){ }^{(18)}$ The volume concentration of formaldehyde in air $(\mathrm{ppm})$ was calculated from $P_{\mathrm{HCHO}}$ by multiplying by $10^{6}$. After exposure to formaldehyde for $1-18 \mathrm{~h}$, the sensor was immersed in an anionic 


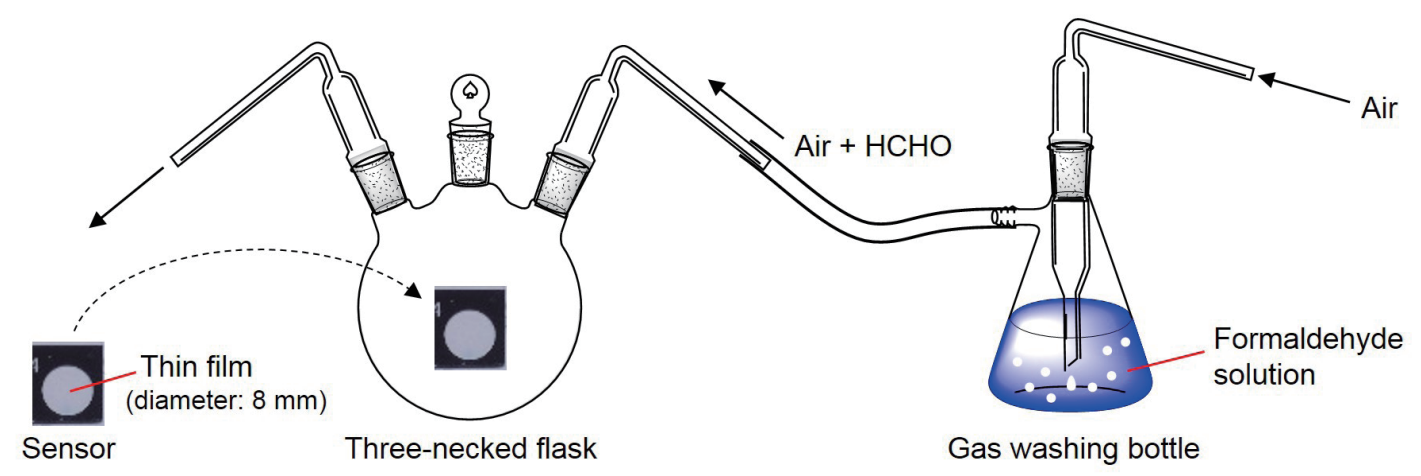

Fig. 2. (Color online) Setup for exposing the sensor to formaldehyde.

dye solution at $25{ }^{\circ} \mathrm{C}$ for 15 min while gently stirring. The dye solution contained $5(50 \mu \mathrm{M}$ for sample A, $30 \mu \mathrm{M}$ for sample $\mathrm{B}$ ) and borate buffer [ $5 \mathrm{mM} \mathrm{B}(\mathrm{OH})_{3}$ and $5 \mathrm{mM} \mathrm{NaBO}_{2} \cdot 4 \mathrm{H}_{2} \mathrm{O}$ ]. Finally, the sensor was washed with water and dried in air, then the UV-vis absorption spectrum of the thin film was measured at normal incidence.

\subsection{Apparatus}

UV-vis absorption spectra were measured using a JASCO V-650 spectrometer equipped with an ISV-722 integrating sphere. Measurements of $\mathrm{pH}$ were carried out using Metrohm $827 \mathrm{pH}$ Lab. Aqueous solutions were prepared using distilled water purified by a Yamato WG202 system.

\section{Results and Discussion}

\subsection{Effect of exposure time to formaldehyde}

To investigate the effect of the exposure time, the formaldehyde sensors were exposed to formaldehyde-containing air for $1-18 \mathrm{~h}$ at $100 \%$ relative humidity $(\mathrm{RH})$ at room temperature. Subsequently, the sensors were immersed in aqueous anionic dye solutions, then the absorption spectra of the thin films in the sensors were measured. Absorbance at $600 \mathrm{~nm}$, which is the maximum absorption wavelength, was plotted against formaldehyde concentration as shown in Fig. 3. It can be seen that some samples were unevenly colored. Since the incident light was set at the center of the circular thin film, the obtained absorbances mainly reflect the color of the central part of the sensor film. When the sensor was exposed for $1 \mathrm{~h}$, it exhibited an apparent color change at $0.75 \mathrm{ppm}$ and became colorless at $1.5 \mathrm{ppm}$. In the case of $3 \mathrm{~h}$ exposure, the sensor showed a visible color change at $0.5 \mathrm{ppm}$. When the exposure time was extended to $18 \mathrm{~h}$, a color change occurred at much lower concentrations: a color change was notable at $0.05 \mathrm{ppm}$ and the sensor was almost colorless at $0.1 \mathrm{ppm}$. These results indicate that the sensitivity of the present formaldehyde sensor strongly depends on the exposure time. It was shown that the 


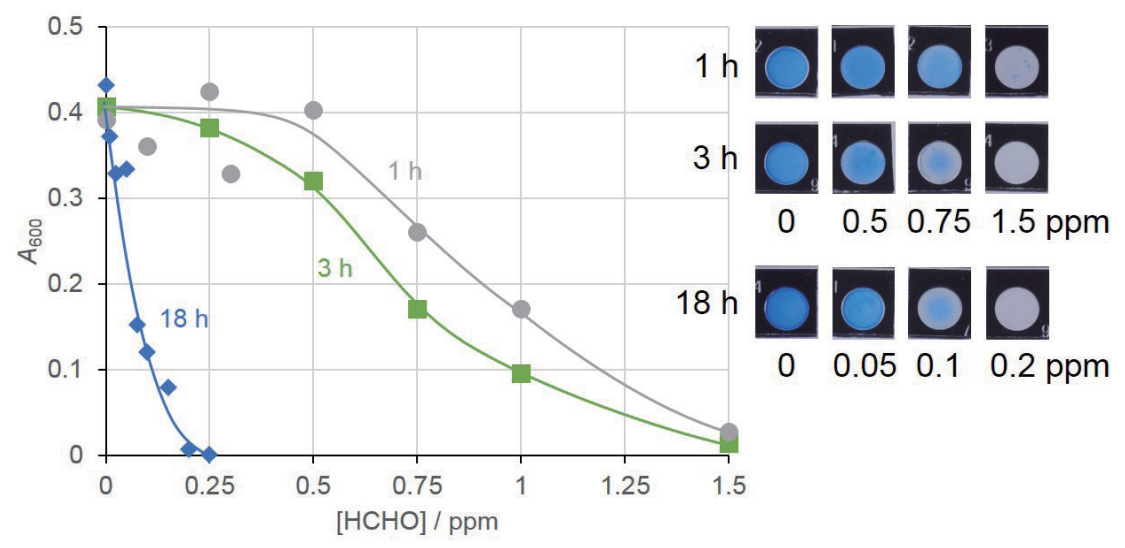

Fig. 3. (Color online) Relationship between formaldehyde concentration and absorbance at $600 \mathrm{~nm}$ (sample A). The exposure times to the formaldehyde-containing air $(100 \% \mathrm{RH})$ were 1,3 , and $18 \mathrm{~h}$.

sensor has the capability (at $100 \% \mathrm{RH}$ ) to measure the indoor guideline level of formaldehyde (0.08 ppm) published by the Japanese Ministry of Health, Labour and Welfare.

\subsection{Effect of humidity}

During the above experiments, we noticed that the sensor response might be dependent on humidity. To confirm this, the humidity of the formaldehyde-containing air was varied by mixing water-vapor-saturated air $(100 \% \mathrm{RH})$ and dry air $(0 \% \mathrm{RH})$. It was found that the formaldehyde sensor showed no response when the RH was $50 \%$ (Fig. 4). We supposed that the wetting condition of the thin film in the sensor has a critically important effect on the sensor responsiveness. At the beginning of the exposure experiment, the thin film was completely dry because it was dried in vacuum before use. Thus, it should have become wet with time upon exposure to the water-vapor-saturated air $(100 \% \mathrm{RH})$ owing to the condensation of water. In contrast, the thin film exposed to the air having $50 \%$ RH was expected to remain dry, indicating that the sensor only works when the thin film is wet. Accordingly, we wetted the film with a small portion of water, then used it for the exposure experiment. However, this trial also resulted in no response at $50 \% \mathrm{RH}$ since initially the wet film soon became dry upon exposure to the air.

To keep the thin film wet throughout the exposure time, we utilized low-volatility solvents such as DMSO. Firstly, the dry film was immersed in DMSO for $1 \mathrm{~h}$, then excess liquid on the film surface was removed by a filter paper. Finally, the thus treated sensor was used for the measurement. In this case, the sensor exhibited clearly visible color changes regardless of humidity (Fig. 5), and the sensitivity at 50\% RH was even higher than that at $100 \% \mathrm{RH}$. The apparent detection limits were 0.3 and $0.2 \mathrm{ppm}$ in the cases of $100 \%$ and $50 \% \mathrm{RH}$, respectively. Comparing these results with those shown in Fig. 4, it is clear that the sensitivity increased for the sensor wetted by DMSO not only at $50 \% \mathrm{RH}$ but also at $100 \% \mathrm{RH}$. The reaction between formaldehyde and a primary amine is accompanied by dehydration. Thus, the formation of the 


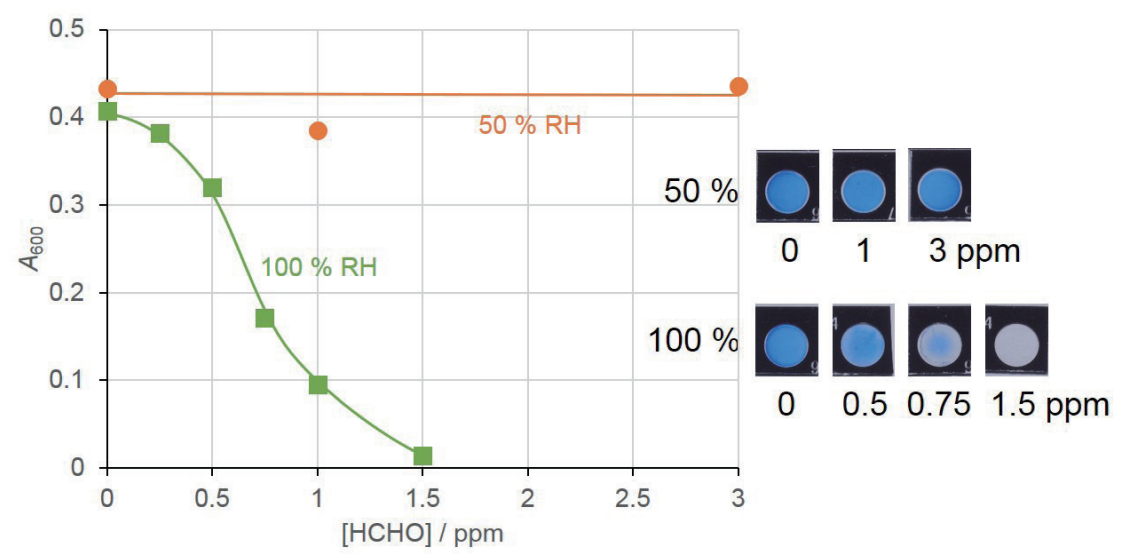

Fig. 4. (Color online) Effect of humidity of formaldehyde-containing air (sample A). The exposure time was $3 \mathrm{~h}$.

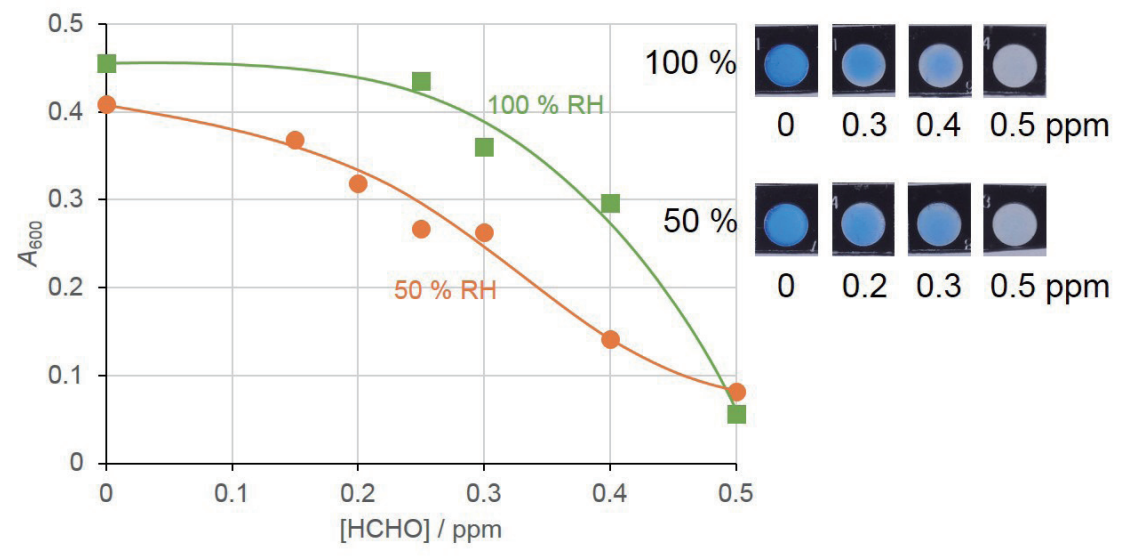

Fig. 5. (Color online) Response of the DMSO-wetted sensors (sample A). The exposure time was $3 \mathrm{~h}$.

imine should more favorably occur in a non-aqueous environment. This is considered to be the reason for the higher sensitivity of the DMSO-wetted samples. These observations suggest that the present method of wetting the thin film with a low-volatility solvent is effective for making the sensor workable under lower humidity conditions.

\subsection{Effect of monomer composition}

To evaluate the effect of the monomer composition on the sensing behavior, we prepared a sensor having a different content of primary amine monomer 1 . The primary amino group in the thin film is thought to be responsible for the color change. Before reacting with formaldehyde, the primary amino groups are positively charged owing to protonation on the nitrogen atom. Thus, anionic dyes are electrostatically adsorbed. After reacting with formaldehyde, the amino groups should lose positive charges because of the formation of uncharged imine groups. Therefore, the sensor gradually becomes colorless by losing its 
affinity to anionic dyes with increasing formaldehyde concentration. A plausible response mechanism is shown in Fig. 6. Because we expected that the sensitivity would increase with increasing primary amine content in the film, the responsiveness of sample A was compared with that of sample B (Fig. 7). It can be clearly seen that sample B with a higher amine content was more sensitive than sample A: sample B exhibited an apparent color change at $0.15 \mathrm{ppm}$ and became colorless at $0.3 \mathrm{ppm}$, whereas sample A became colorless at $0.5 \mathrm{ppm}$. As shown in Table 1, sample B contains a 1.7 times higher concentration of the primary amino group. Therefore, a formaldehyde molecule has more opportunities to encounter an amino group in sample B, resulting in a higher sensitivity.
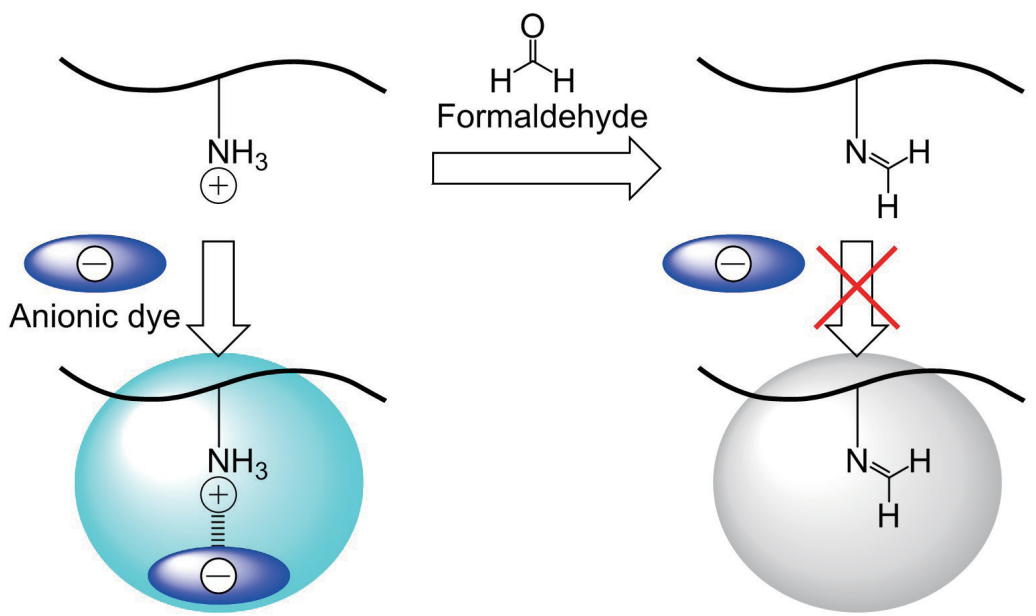

Fig. 6. (Color online) Mechanism of formaldehyde-responsive color change in thin film.

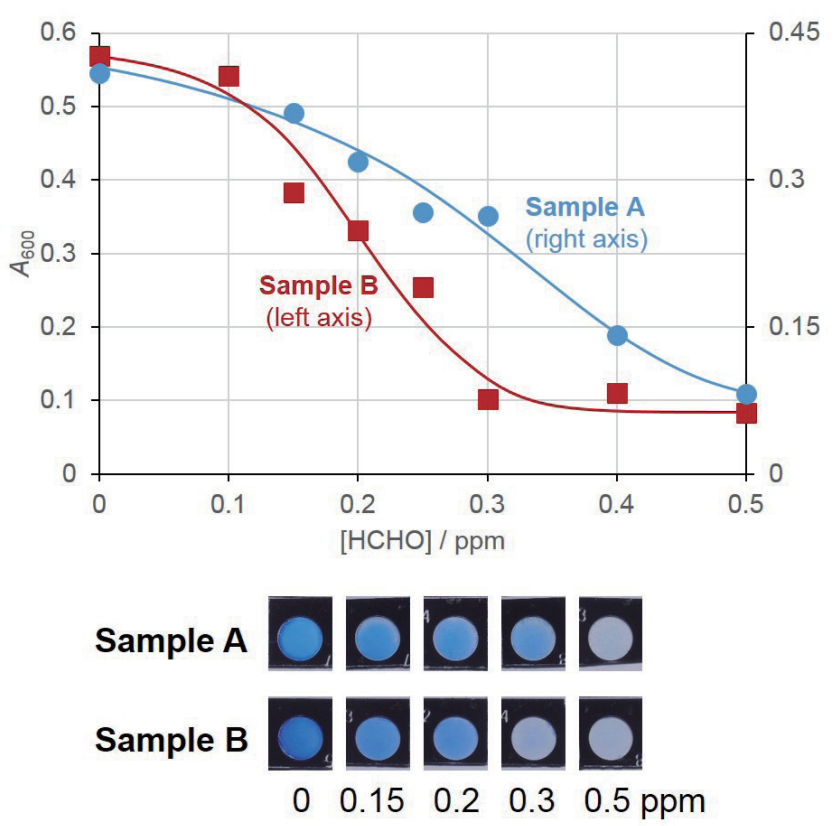

Fig. 7. (Color online) Effect of monomer composition on sensor responsiveness (50\% RH). The exposure time was $3 \mathrm{~h}$. 


\section{Conclusions}

We have developed a novel methodology for sensing formaldehyde in air that displays its response as distinct color changes. By copolymerizing a primary amine monomer with other co-monomers on a glass substrate, a formaldehyde sensor composed of a primary-amine-containing thin film was prepared. After exposure to formaldehyde-containing air, the thin film was immersed in an anionic dye solution. It was demonstrated that the color of the thin film changed in accordance with the formaldehyde concentration in air. We found that the sensing behavior was dependent on the humidity of the air, and the sensitivity was reduced when the humidity was low. To overcome this problem, we used a low-volatility solvent to wet the thin film. This approach resulted in successful sensing at a lower humidity. We also found that the sensitivity was increased by increasing the primary amine content in the thin film. These findings provide a rational way to develop sensitive and user-friendly versatile sensors for formaldehyde.

The formaldehyde sensor presented herein has the following advantages: 1) it is prepared by common and low-cost chemicals, and its preparation does not require complex organic synthesis and manufacturing processes, 2) a wide range of anionic dyes are applicable, so one can choose any dye having a desirable color with low toxicity and low cost. We believe that the present methodology will be the basis for the development of practical sensors for gaseous formaldehyde in the near future.

\section{Acknowledgments}

This work was supported by a grant-in-aid from Northern Advancement Center for Science and Technology, Hokkaido, Japan.

\section{References}

1 T. Takigawa, B. L. Wang, Y. Saijo, K. Morimoto, K. Nakayama, M. Tanaka, E. Shibata, T. Yoshimura, H. Chikara, K. Ogino, and R. Kishi: Int. Arch. Occup. Environ. Health 83 (2010) 225. https://doi.org/10.1007/ s00420-009-0475-9

2 G. D. Nielsen, S. T. Larsen, and P. Wolkoff: Arch. Toxicol. 87 (2013) 73. https://doi.org/10.1007/s00204-0120975-3

3 M. D. Colton, P. MacNaughton, J. Vallarino, J. Kane, M. Bennett-Fripp, J. D. Spengler, and G. Adamkiewicz: Environ. Sci. Technol. 48 (2014) 7833. https://doi.org/10.1021/es501489u

4 B. Glas, B. Stenberg, H. Stenlund, and A. L. Sunesson: Int. Arch. Occup. Environ. Health 88 (2015) 613. https://doi.org/10.1007/s00420-014-0985-y

5 H. Zhu, J. She, M. Zhou, and X. Fan: Sens. Actuators, B 283 (2019) 182. https://doi.org/10.1016/j.snb.2018.11.156

6 M. N. Descamps, T. Bordy, J. Hue, S. Mariano, G. Nonglaton, E. Schultz, T. H. Tan-Thi, and S. Vignoud-Despond: Sens. Actuators, B 170 (2012) 104. https://doi.org/10.1016/j.snb.2011.02.032

7 H. Li, Y. Li, M. Li, L. Xu, and J. Li: Sens. Actuators, B 284 (2019) 657. https://doi.org/10.1016/j.snb.2019.01.021

8 S. Ishihara, J. Labuta, T. Nakanishi, T. Tanaka, and H. Kataura: ACS Sens. 2 (2017) 1405. http://dx.doi. org/10.1021/acssensors.7b00591

9 Y. Yamada-Maruo, J. Nakamura, and M. Uchiyama: Talanta 74 (2008) 1141. https://doi.org/10.1016/ j.talanta.2007.08.017

10 X. Wang, Y. Si, X. Mao, Y. Li, J. Yu, H. Wang, and B. Ding: Analyst 138 (2013) 5129. https://doi.org/10.1039/ C3AN00812F 
11 X. Wang, Y. Li, X. Li, J. Yu, S. S. Al-Deyab, and B. Ding: Sens. Actuators, B 203 (2014) 333. https://doi. org/10.1016/j.snb.2014.06.101

12 J. Li, C. Hou, D. Huo, M. Yang, H.-B. Fa, and P. Yang: Sens. Actuators, B 196 (2014) 10. https://doi. org/10.1016/j.snb.2014.01.054

13 Y. Iwami, T. Yokozawa, W. Takayoshi, and Y. Kanekiyo: Talanta 85 (2011) 829. https://doi.org/10.1016/ j.talanta.2011.04.068

14 Y. Iwami, H. Yamamoto, and Y. Kanekiyo: Chem. Lett. 42 (2013) 1214. https://doi.org/10.1246/cl.130599

15 W. Takayoshi, M. Imajo, M. Iijima, M. Suzuki, H. Yamamoto, and Y. Kanekiyo: Sens. Actuators, B 192 (2014) 776. https://doi.org/10.1016/j.snb.2013.11.008

16 Y. Iwami, T. Yokozawa, H. Yamamoto, and Y. Kanekiyo: J. Appl. Polym. Sci. 132 (2015) 42679. https://doi. org/10.1002/app.42679

17 T. Denda, R. Mizutani, M. Iijima, H. Nakahashi, H. Yamamoto, and Y. Kanekiyo: Talanta 144 (2015) 816. https://doi.org/10.1016/j.talanta.2015.06.012

18 R. Seyfioglu and M. Odabasi: Environ. Monit. Assess. 128 (2007) 343. https://doi.org/10.1007/s10661-0069317-3

\section{About the Authors}

Yasumasa Kanekiyo received his B.S. and M.S. degrees from Nagoya University, Japan, in 1993 and 1995, respectively, and his Ph.D. degree from Kyushu University, Japan, in 2001. Since 2006, he has been an associate professor at Kitami Institute of Technology, Japan. His research interests are in molecular recognition chemistry, molecular machines, and sensing systems utilizing them. (kanekiyo@mail.kitami-it.ac.jp)

You Mitani received his B.S. from Kitami Institute of Technology in 2018. Since 2018, he has been a graduate student at Kitami Institute of Technology.

Miho Suda was an assistant researcher at Kitami Institute of Technology from 2018 to 2019.

Hiroshi Aoki received his B.S., M.S., and Ph.D. degrees from The University of Tokyo (UT) in 1996, 1998, and 2001, respectively. From 2001 to 2004, he worked as a postdoctoral fellow at UT and National Institute of Advanced Industrial Science and Technology (AIST). Since 2004, he has been a research scientist and a senior research scientist at AIST. His research interests are in simple and rapid oligonucleotide sensing and its applications to the evaluation of chemical toxicity. (aoki-h@aist.go.jp)

Hironobu Minai received his B.S. degree from Kanagawa Institute of Technology, Japan, in 2002. Since 2002, he has been working in JOKOH Co., Ltd., Tokyo, Japan.

(h_minai@jokoh.com) 